\title{
CHANGES OF VARIABLES NEAR A PERIODIC SURFACE OR INVARIANT MANIFOLD
}

\author{
$\stackrel{\text { BY }}{\text { AL }}$ KELLEY(1)
}

1. Introduction. For a certain class of ordinary differential equations (see (1) below) we construct analytic changes of variables which reduce the system (parallelize the flow near the periodic surface) in such a way that the results of [5] apply in order to linearize (in general) the original system. Thus these linearization results (Theorems 2 and 3 below) depend on both Theorem 1 below and the results of [5]. The essential new technique used in the proof of Theorem 1 is exhibited by solving equation (6) below. Other variations of this technique have proved useful in [6] and [7] also.

Generalizing a well-known linearization result due to C. L. Siegel [8], Belaga [1] has proved a linearization theorem which contains our Theorems 1 and 2 below as a special case when the matrix $A$ in (1) below is diagonal. However, because we make the stronger restriction that the eigenvalues of $A$ all have negative real parts, our proof is different and simpler $i$ that it does not require the technique of quadratic convergence. Moreover our theorems extend immediately to the cases of almost periodic $\theta$-dependence and bounded $\theta$-dependence (see Remarks 1 and 2 below).

This paper constitutes in part the author's thesis written under the direction of Professor S. P. Diliberto, and many of the underlying ideas have their origin in [2], [3], [4]. In particular, equation (17) below is to be found in [4].

2. Notation. The norm $|\cdot|$ will represent the euclidean norm on vectors and the operator norm on matrices. On pairs of vectors $\langle\cdot, \cdot\rangle$ will represent the usual (complex) inner product. If $G=G(g)$ is a smooth, vector valued function of the vector $g$, then $G_{g}$ will designate the Jacobian matrix of partial derivatives. In addition $D_{g}^{\rho}$ will designate the partial differential operator

$$
\partial^{|\rho|} / \partial g_{1}^{\rho_{1}} \ldots \partial g_{n^{n}}^{\rho^{n}}
$$

where $n=\operatorname{dim} g, \rho=\left(\rho_{1}, \ldots, \rho_{n}\right)$ is an $n$-tuple of nonnegative integers, $|\rho|=\rho_{1}+$ $\cdots+\rho_{n}$ (the "norm" here is not euclidean which we justify by not considering $\rho$ as a vector).

Let $p$ and $q$ be complex vectors (dimensions of $p$ and $q$ unspecified) and let $\nu$ be a nonnegative integer. We define $\Gamma_{a}^{v}(p: q)$ to be the class of functions $\{F\}$ which satisfy (i), (ii), (iii) below.

Received by the editors December 5, 1966 and, in revised form, February 9, 1967.

(1) This work was supported in part by the Office of Naval Research. 
(i) $F=F(p, q)$ is a complex vector valued or matrix valued function ( $\operatorname{dim} F$ is not necessarily equal to $\operatorname{dim} G$ even though $F, G \in \Gamma_{a}^{v}(p: q)$ ) defined and analytic on

$$
N_{o}=\{(p, q)|| \mathscr{I}(p)|<\delta,| q \mid<\delta\},
$$

for some $\delta>0$, where $\mathscr{I} \equiv$ imaginary part, $\mathscr{I}(p)=\left(\mathscr{I}\left(p_{1}\right), \mathscr{I}\left(p_{2}\right), \ldots, \mathscr{I}\left(p_{m}\right)\right)$ if $m=\operatorname{dim} p$. The positive constant $\delta$ depends on $F$.

(ii) $F$ has a convergent power series expansion in $q$ (components of $q$ ) about the origin $q=0$; the coefficients in the expansion are bounded, analytic, vector valued or matrix valued (as the case may be) functions of $p$ for $p \in\{p|| \mathscr{I}(p) \mid<\delta\}$; there are no terms of degree less than $\nu$ in the expansion of $F$.

(iii) For some $K>0$ the components of $F$ are majorized by

$$
K\left(q_{1}+\cdots+q_{n}\right)^{v}\left[1-K\left(q_{1}+\cdots+q_{n}\right)\right]^{-1},
$$

uniformly in $p=\{p|| \mathscr{I}(p) \mid<\delta\}$ where $n=\operatorname{dim} q$. The constant $K$ depends on $F$.

Let $p$ be a real vector and $q$ be a complex vector. In an analogous way we define $\Gamma^{\nu}(p: q)$ to be the class of functions $\{G\}$ such that

(i) $G=G(p, q)$ is a complex vector valued or matrix valued function defined and continuous on

$$
\tilde{N}_{\delta}=\left\{(p, q)\left|p \in R^{m},\right| q \mid<\delta\right\},
$$

for some $\delta>0$, where $m=\operatorname{dim} p$ and $R^{m}$ is real euclidean $m$-space.

(ii) $G$ has a convergent power series expansion in $q$ about $q=0$; the coefficients in the expansion are bounded, continuous vector valued or matrix valued (as the case may be) functions of $p$ for $p \in R^{m}$; there are no terms of degree less than $\nu$ in the expansion of $G$.

(iii) For some $K>0$ the components of $G$ are majorized by

$$
K\left(q_{1}+\cdots+q_{n}\right)^{v}\left[1+K\left(q_{1}+\cdots+q_{n}\right)\right]^{-1},
$$

uniformly in $p \in R^{m}$ where $n=\operatorname{dim} q$. The constant $K$ depends on $G$.

3. Changes of variables. Consider the analytic system of ordinary differential equations

$$
\dot{\theta}=a+\Theta(\theta, x), \quad \dot{x}=A x+X(\theta, x),
$$

where $a, \theta, \Theta$ are $m$-vectors; $x$ and $X$ are $n$-vectors; $a$ is a real, constant vector ( $a=0$ is allowed); $A$ is an $n \times n$ constant matrix in Jordan canonical form; $A=\operatorname{diag}\left(\lambda_{1}, \ldots, \lambda_{n}\right)+\operatorname{subdiag}\left(\gamma_{1}, \ldots, \gamma_{n-1}\right)$ where $\gamma_{j}=0$ or $\gamma$ with $\gamma$ a sufficiently small positive constant (to be specified below); the eigenvalues of $A$ all have negative real parts; $\Theta \in \Gamma_{a}^{1}(\theta: x) ; X \in \Gamma_{a}^{2}(\theta: x) ; \Theta$ and $X$ have real multiple period $\omega$ in $\theta$.

THEOREM 1. For system (1) there exists a unique change of variables

$$
\varphi=\theta-\Phi(\theta, x)
$$


such that (1) transforms to

$$
\dot{\varphi}=a, \quad \dot{x}=A x+\tilde{X}(\varphi, x),
$$

where $\Phi \in \Gamma_{a}^{1}(\theta: x)$; $\Phi$ has multiple period $\omega$ in $\theta ; \tilde{X}(\varphi, x)=X(\theta, x)$ on an appropriate (new) $N_{\delta}, \varphi$ and $\theta$ being related by (2).

Proof. Assuming such a change of variables exists, we take the derivative of both sides of (2),

$$
\dot{\varphi}=\dot{\theta}-\dot{\Phi}
$$

which then yields from (1) and (3)

$$
a=a+\Theta(\theta, x)-\dot{\Phi}(\theta, x), \quad \dot{\Phi}(\theta, x)=\Theta(\theta, x) .
$$

Let $(\psi(t), \xi(t))$ with $\psi(t)=\psi(t, \theta, x), \xi(t)=\xi(t, \theta, x)$, represent the unique solution of (1) with initial condition $(\theta, x)$ at $t=0$. Equation (4) has two interpretations. First we may take the derivative with respect to $t$ implicitly,

$$
\Phi_{\theta} \dot{\theta}+\Phi_{x} \dot{x}=\Theta
$$

and then from (1) we obtain

$$
\Phi_{\theta}(\theta, x)\{a+\Theta(\theta, x)\}+\Phi_{x}(\theta, x)\{A x+X(\theta, x)\}=\Theta(\theta, x) .
$$

Secondly, the derivative with respect to $t$ may be taken directly when $(\theta, x)$ in (4) is replaced by solution curves $(\psi(t), \xi(t))$;

$$
\frac{d}{d t} \Phi(\psi(t), \xi(t))=\Theta(\psi(t), \xi(t))
$$

To solve (6) define $\Phi$ by the equation

$$
\Phi(\theta, x)=\int_{+\infty}^{0} \Theta(\psi(\sigma, \theta, x), \xi(\sigma, \theta, x)) d \sigma
$$

Assuming for the moment that the integral on the right side of (7) exists, we wish to show that $\Phi(\psi(t), \xi(t))$ actually does satisfy (6). Since (1) is an autonomous system,

$$
\begin{aligned}
& \psi(\sigma, \psi(t, \theta, x), \xi(t, \theta, x))=\psi(t+\sigma, \theta, x) \\
& \xi(\sigma, \psi(t, \theta, x), \xi(t, \theta, x))=\xi(t+\sigma, \theta, x)
\end{aligned}
$$

so that

$$
\Phi(\psi(t), \xi(t))=\Phi(\psi(t, \theta, x), \xi(t, \theta, x))=\int_{+\infty}^{0} \Theta(\psi(t+\sigma, \theta, x), \xi(t+\sigma, \theta, x)) d \sigma .
$$

Setting $t+\sigma=\tau$, we obtain

$$
\Phi(\psi(t), \xi(t))=\int_{+\infty}^{t} \Theta(\psi(\tau), \xi(\tau)) d \tau
$$

and clearly $\Phi$ will satisfy (6).

Let us now show that the integral on the right side of (7) exists and that 
$\Phi \in \Gamma_{a}^{1}(\theta: x)$. Since $A$ is a contraction matrix $\left(\mathscr{R}\left(\lambda_{j}\right)<0,(j=1, \ldots, n), \mathscr{R} \equiv\right.$ real part $)$ in Jordan canonical form, there exists $\mu>0$ such that

$$
\mathscr{R}(\langle A x, x\rangle) \leqq-2 \mu|x|^{2}
$$

holds for all (complex) $n$-vectors $x$. In particular $-2 \mu$ may be chosen as

$$
\frac{1}{2} \max _{1 \leqq j \leqq n} \mathscr{R}\left(\lambda_{j}\right),
$$

provided that the off-diagonal components of $A$ are sufficiently small

$$
\left(0<\gamma \leqq \frac{1}{2}(n-1)^{-1}\left|\max _{1 \leqq i \leqq n} \mathscr{R}\left(\lambda_{j}\right)\right| \text { suffices }\right) \text {. }
$$

By hypothesis $\Theta \in \Gamma_{a}^{1}(\theta: x)$ and $X \in \Gamma_{a}^{2}(\theta: x)$ so that for some $\delta>0$ both $\Theta$ and $X$ are defined and analytic on

$$
N_{\delta}=\{(\theta, x)|| \mathscr{I}(\theta)|<\delta,| x \mid<\delta\} .
$$

By restricting $\delta$ further, if necessary, we may assume that

$$
|X(\theta, x)| \leqq \mu|x|
$$

holds for all $(\theta, x) \in N_{\delta}$. From (1), (8), (9) we obtain

$$
\begin{aligned}
\frac{d}{d t}|\xi(t)|^{2} & =\langle\dot{\xi}(t), \xi(t)\rangle+\langle\xi(t), \dot{\xi}(t)\rangle=2 \mathscr{R}(\langle\dot{\xi}(t), \xi(t)\rangle) \\
& =2 \mathscr{R}(\langle A \xi(t), \xi(t)\rangle)+2 \mathscr{R}(\langle X(\psi(t), \xi(t)), \xi(t)\rangle) \\
& \leqq-4 \mu|\xi(t)|^{2}+2|X(\psi(t), \xi(t))||\xi(t)| \\
& \leqq-4 \mu|\xi(t)|^{2}+2 \mu|\xi(t)|^{2} \leqq-2 \mu|\xi(t)|^{2},
\end{aligned}
$$

provided $(\psi(t), \xi(t)) \in N_{\delta}$. If we choose initial conditions $(\theta, x) \in N_{\delta}$, then there exists $T=T(\theta, x), T>0$, such that $(\psi(t), \xi(t)) \in N_{\delta}$ for $0 \leqq t \leqq T$. Thus, from (10) for $(\theta, x) \in N_{\delta}$ and $0 \leqq t \leqq T(\theta, x)$ we have

$$
|\xi(t, \theta, x)| \leqq e^{-\mu t}|x|,
$$

and therefore $|\xi(t)| \downarrow 0$ as long as $|\mathscr{I}(\psi(t))|<\delta$. Since $\Theta \in \Gamma_{a}^{1}(\theta: x)$, for some $K>0$ sufficiently large

$$
|\Theta(\theta, x)| \leqq K|x|
$$

for all $(\theta, x) \in N_{\delta}$. For $t \geqq 0$ we have from (1), (11), (12),

$$
\begin{aligned}
\psi(t, \theta, x) & =\theta+a t+\int_{0}^{t} \Theta(\psi(\tau, \theta, x), \xi(\tau, \theta, x)) d \tau, \\
|\mathscr{I}(\psi(t, \theta, x))| & \leqq|\mathscr{I}(\theta)|+K \int_{0}^{t}|\xi(\tau, \theta, x)| d \tau, \\
|\mathscr{I}(\psi(t, \theta, x))| & \leqq|\mathscr{I}(\theta)|+K \int_{0}^{t} e^{-\mu \tau}|x| d \tau, \\
|\mathscr{I}(\psi(t, \theta, x))| & \leqq|\mathscr{I}(\theta)|+K \mu^{-1}|x|,
\end{aligned}
$$


as long as $(\psi(t), \xi(t)) \in N_{\delta}$. By choosing $\delta_{1}$ sufficiently small, $0<\delta_{1} \leqq \delta$, it follows from (11) and (13) that

$$
(\psi(t, \theta, x), \xi(t, \theta, x)) \in N_{\delta}, \quad 0 \leqq t<\infty,
$$

provided $(\theta, x) \in N_{\delta_{1}}$. Using (11) and (12) in (7) it follows readily that $\Phi$ is defined in $N_{\delta_{1}}$. Since (1) is an analytic system of differential equations, $\psi(t, \theta, x)$ and $\xi(t, \theta, x)$ are analytic in $(t, \theta, x)$, and hence $\Phi$ is analytic in $N_{\delta_{1}}$ and $\Phi \in \Gamma_{a}^{1}(\theta: x)$. Using a standard argument, one shows from (7) that $\Phi$ has multiple period $\omega$ in $\theta$; therefore to prove Theorem 1 all that remains to be shown is the uniqueness of $\Phi$.

Let $A=A^{0}+A^{1}$ where $A^{0}=\operatorname{diag}\left(\lambda_{1}, \ldots, \lambda_{n}\right)$ and $A^{1}=\operatorname{subdiag}\left(\gamma_{1}, \ldots, \gamma_{n-1}\right)$. From (5) we have

$$
\Phi_{\theta} a+\Phi_{x} A^{0} x=\Theta-\Phi_{\theta} \Theta-\Phi_{x} A^{1} x-\Phi_{x} X
$$

Let

$$
\begin{array}{ll}
\Theta_{j}(\theta, x)=\sum_{|\alpha| \geqq 1} \Theta_{j, \alpha}(\theta) x^{\alpha} \quad(j=1, \ldots, m), \\
X_{j}(\theta, x)=\sum_{|\alpha| \geqq 2} X_{j, \alpha}(\theta) x^{\alpha} \quad(j=1, \ldots, n), \\
\Phi_{j}(\theta, x)=\sum_{|\alpha| \geqq 1} \Phi_{j, \alpha}(\theta) x^{\alpha} \quad(j=1, \ldots, m),
\end{array}
$$

where $\alpha=\left(\alpha_{1}, \ldots, \alpha_{n}\right)$ is an $n$-tuple of nonnegative integers; $|\alpha|=\alpha_{1}+\cdots+\alpha_{n}$; $x^{\alpha}=x_{1}^{\alpha} \cdots x_{n}^{\alpha}$.

From (14)

$$
\begin{aligned}
\sum_{l=1}^{m} a_{l} \frac{\partial}{\partial \theta_{l}} \Phi_{j}+\sum_{l=1}^{n} \lambda_{l} x_{l} \frac{\partial}{\partial x_{l}} \Phi_{j}= & \Theta_{j}-\sum_{l=1}^{m} \Theta_{l} \frac{\partial}{\partial \theta_{l}} \Phi_{j}-\sum_{l=2}^{n} \gamma_{l-1} x_{l-1} \frac{\partial}{\partial x_{l}} \Phi_{j} \\
& -\sum_{l=1}^{n} X_{l} \frac{\partial}{\partial x_{l}} \Phi_{j} \quad(j=1, \ldots, m) .
\end{aligned}
$$

Upon equating coefficients of $x^{\alpha}$ on both sides of (15), we obtain

$$
\sum_{l=1}^{m} a_{l} \frac{\partial}{\partial \theta_{l}} \Phi_{j, \alpha}+\left[\sum_{l=1}^{n} \alpha_{l} \lambda_{l}\right] \Phi_{j, \alpha}=Z_{j, \alpha}
$$

where $Z_{j, \alpha}=Z_{j, \alpha}(\theta)$ is the coefficient of $x^{\alpha}$ on the right side of (15). Define $\lambda_{\alpha}=\sum_{l=1}^{n} \alpha_{l} \lambda_{l}$.

Since $A$ is a contraction matrix, $\mathscr{R}\left(\lambda_{\alpha}\right)<0$ for all $|\alpha|>0$. If we suppose that $Z_{j, \alpha}(\theta)$ is a known function defined and analytic on $\{\theta|| \mathscr{I}(\theta) \mid<\delta\}$ and has multiple period $\omega$ in $\theta$, then (16) has a unique solution defined and analytic on $\{\theta|| \mathscr{I}(\theta) \mid<\delta\}$ with multiple period $\omega$ in $\theta$, this solution being given by (recall that $a$ is a real constant vector)

$$
\Phi_{j, \alpha}(\theta)=\int_{+\infty}^{0} e^{\lambda_{\alpha} \sigma} Z_{j, \alpha}(\theta+a \sigma) d \sigma
$$


Using formula (17), one can compute the coefficients of $\Phi(\theta, x)$ recursively by following along the proper order relation defined on the subscripts $(j, \alpha)$. See [5] for details. Thus $\Phi(\theta, x)$ is unique and the proof of Theorem 1 is complete.

REMARK 1. Observe that multiple periodicity is not essential. If $\Theta, \Theta_{\theta} \in \Gamma_{a}^{1}(\theta: x)$; $X, X_{\theta} \in \Gamma_{a}^{2}(\theta: x)$; then the above arguments yield a unique change of variables $\varphi=\theta-\Phi(\theta, x)$ such that $\dot{\varphi}=a$ where $\Phi, \Phi_{\theta} \in \Gamma_{a}^{1}(\theta: x)$. In addition if $\Theta$ and $X$ are almost periodic in $\theta$ when $\theta$ is restricted to be real, then $\Phi$ will also be almost periodic in $\theta$ for real $\theta$, and $\Phi$ will have the same frequencies as $\Theta$ and $X$.

We now assume with no loss of generality that the eigenvalues of $A$ have the following ordering,

$$
\mathscr{R}\left(\lambda_{n}\right) \leqq \mathscr{R}\left(\lambda_{n-1}\right) \leqq \cdots \leqq \mathscr{R}\left(\lambda_{1}\right)<0 .
$$

As before let $\alpha=\left(\alpha_{1}, \ldots, \alpha_{n}\right)$ represent $n$-tuples of nonnegative integers and let $|\alpha|=\alpha_{1}+\cdots+\alpha_{n}$. Let $\left(C_{j}\right), 1 \leqq j \leqq n$, represent the following condition on $\alpha$,

$$
\left(C_{j}\right): \mathscr{R}\left[\sum_{l=1}^{n} \alpha_{l} \lambda_{l}-\lambda_{j}\right] \neq 0 .
$$

Notice that (18) implies $\left(C_{1}\right)$ holds for all $|\alpha|>1$.

THEOREM 2. If conditions $\left(C_{j}\right)(j=2, \ldots, n)$ hold for all $|\alpha|>1$, then for system (1) with (18) there exists a unique change of variables

$$
p=x-P(\theta, x)
$$

such that (1) transforms to

$$
\dot{\theta}=a+\tilde{\Theta}(\theta, p), \quad \dot{p}=A p,
$$

where $P \in \Gamma_{a}^{2}(\theta: x) ; P$ has multiple period $\omega$ in $\theta ; \tilde{\Theta}(\theta, p)=\Theta(\theta, x)$ on an appropriate (new) $N_{\delta}, p$ and $x$ being related by (19).

Proof. Suppose that $P$ exists as a formal power series with undetermined coefficients,

$$
P_{j}(\theta, x)=\sum_{|\alpha|>1}^{\infty} P_{j, \alpha}(\theta) x^{\alpha} \quad(j=1, \ldots, n) .
$$

Differentiating both sides of (19), it follows from (1), (19), (20) that

$$
\begin{gathered}
P_{\theta}\{a+\Theta\}+P_{x}\{A x+X\}-A P=X, \\
P_{\theta} a+P_{x} A^{0} x-A^{0} P=A^{1} P+X-P_{\theta} \Theta-P_{x} A^{1} x-P_{x} X,
\end{gathered}
$$

or equivalently,

$$
\begin{aligned}
\sum_{l=1}^{m} a_{l} \frac{\partial}{\partial \theta_{l}} P_{j}+\sum_{l=1}^{n} \lambda_{l} x_{l} & \frac{\partial}{\partial x_{l}} P_{j}-\lambda_{j} P_{j}=\gamma_{j-1} P_{j-1}+X_{j}-\sum_{l=1}^{m} \Theta_{l} \frac{\partial}{\partial \theta_{l}} P_{j} \\
& -\sum_{l=2}^{n} \gamma_{l-1} x_{l-1} \frac{\partial}{\partial x_{l}} P_{j}-\sum_{l=1}^{n} X_{l} \frac{\partial}{\partial x_{l}} P_{j} \quad(j=1, \ldots, n)
\end{aligned}
$$


where, of course, $\gamma_{0}$ is defined to be zero. Equating coefficients of $x^{\alpha}$ on both sides of (21), we have

$$
\sum_{l=1}^{m} a_{l} \frac{\partial}{\partial \theta_{l}} P_{j, \alpha}+\lambda_{j, \alpha} P_{j, \alpha}=Z_{j, \alpha}
$$

where

$$
\lambda_{j, \alpha}=\sum_{l=1}^{n} \alpha_{l} \lambda_{l}-\lambda_{j}
$$

and $Z_{j, \alpha}=Z_{j, \alpha}(\theta)$ is the coefficient of $x^{\alpha}$ on the right side of (21). If we suppose that $Z_{j, \alpha}(\theta)$ is a known function defined and analytic on $\{\theta|| \mathscr{I}(\theta) \mid<\delta\}$ and has multiple period $\omega$ in $\theta$, then (22) has a unique solution defined and analytic on $\{\theta|| \mathscr{I}(\theta) \mid<\delta\}$ with multiple period $\omega$ in $\theta$, this solution being given by

$$
P_{j, \alpha}(\theta)=\int_{ \pm \infty}^{0} e^{\lambda_{j, \alpha}{ }^{\circ}} Z_{j, \alpha}(\theta+a \sigma) d \sigma
$$

where the lower limit on the integral is chosen as $+\infty$ if $\mathscr{R}\left(\lambda_{j, \alpha}\right)<0$ and $-\infty$ if $\mathscr{R}\left(\lambda_{j, \alpha}\right)>0$. The hypothesis that conditions $\left(C_{j}\right)(j=2, \ldots, n)$ hold for all $|\alpha|>1$ implies that $\mathscr{R}\left(\lambda_{j, \alpha}\right) \neq 0$. Using formula (23), the coefficients of $P(\theta, x)$ can be computed recursively by following along the proper order relation (see [5]) defined on the subscripts $(j, \alpha)$. Thus $P(\theta, x)$ is uniquely defined as a formal power series. To prove convergence we would like to use the Cauchy majorant method developed in [5]. However, the terms $\Theta_{l}\left(\partial / \partial \theta_{l}\right) P_{j}$ in the right side of (21) prevent us from doing this. There seems to be no way of (directly) constructing a majorant for $\left(\partial / \partial \theta_{l}\right) P_{j}$. To get around this difficulty, introduce the change of variables (2) so that system (1) transforms to (3). Now we look for a change of variables

$$
p=x-\tilde{P}(\varphi, x)
$$

such that (3) reduces to

$$
\dot{\varphi}=a, \quad \dot{p}=A p .
$$

Differentiating both sides of (24), we have from (3), (24), (25) that

$$
\begin{aligned}
\sum_{l=1}^{m} a_{l} \frac{\partial}{\partial \varphi_{l}} \tilde{P}_{j}+\sum_{l=1}^{n} \lambda_{l} x_{l} & \frac{\partial}{\partial x_{l}} \tilde{P}_{j}-\lambda_{j} \tilde{P}_{j}=\gamma_{j-1} \tilde{P}_{j-1}+\tilde{X}_{j} \\
& -\sum_{l=2}^{n} \gamma_{l-1} x_{l-1} \frac{\partial}{\partial x_{l}} \tilde{P}_{j}-\sum_{l=1}^{n} X_{l} \frac{\partial}{\partial x_{l}} \tilde{P}_{j} \quad(j=1, \ldots, n) .
\end{aligned}
$$

Proceeding as before, we can compute $\tilde{P}(\varphi, x)$ as a formal power series. But in addition, since there are no terms $\left(\partial / \partial \varphi_{l}\right) \widetilde{P}_{j}$ in the right side of (26), we can follow [5] (in particular, the proof of Theorem 1 in [5]) to construct a majorant for $\tilde{P}$. Thus $\widetilde{P} \in \Gamma_{a}^{2}(\varphi: x)$, and we conclude that

$$
P(\theta, x)=\tilde{P}(\theta-\Phi(\theta, x), x)
$$


is defined and analytic on $N_{\delta_{2}}$ for some $\delta_{2}>0$ sufficiently small. This completes the proof of Theorem 2.

REMARK 2. Again we observe that multiple periodicity is not essential to the above arguments.

THEOREM 3. For system (1) with (18) there exists a change of variables

$$
\varphi=\theta-\Phi(\theta, x), \quad p=x-P(\theta, x),
$$

not necessarily unique, such that (1) transforms to

$$
\dot{\varphi}=a, \quad \dot{p}=A p+\hat{P}(\varphi, p),
$$

where $\Phi \in \Gamma_{a}^{1}(\theta: x) ; P \in \Gamma_{a}^{2}(\theta: x) ; \hat{P} \in \Gamma_{a}^{2}(\varphi: p) ; \Phi$ and $P$ have multiple period $\omega$ in $\theta ; \hat{P}$ has multiple period $\omega$ in $\varphi$; the components of $\hat{P}=\left(\hat{P}_{1}, \ldots, \hat{P}_{n}\right)$ are polynomials of the form

$$
\begin{aligned}
\hat{P}_{1} & \equiv 0 \\
\hat{P}_{j}(\varphi, p) & =\sum \hat{P}_{j, \alpha_{1}, \ldots, \alpha_{y-1}}(\varphi) p_{1}^{\alpha_{1}} \cdots p_{j-1}^{\alpha_{j}-1} \quad(j=2, \ldots, n)
\end{aligned}
$$

the summation in (29) being taken over all $|\alpha|>1$ which do not satisfy condition $\left(C_{j}\right)$, in particular if conditions $\left(C_{j}\right)(j=2, \ldots, n)$ hold for all $|\alpha|>1$, then $\widetilde{P} \equiv 0$ and the change of variables (27) is unique.

Proof. The proof of Theorem 3 is similar to the proof of Theorem 2. First the change of variables (2) is used to reduce (1) to system (3). Then the proof method of Theorem 2 in [5] is used to reduce (3) to system (28). This completes our proof.

Remark 3. Observe that Theorem 3 includes Theorem 2 as a special case. Nevertheless, we have included Theorem 2 in order to point out that when all the conditions $\left(C_{j}\right)(j=2, \ldots, n)$ hold, then $P$ in (19) and (27) is readily computable as a power series with multiply periodic coefficients. When the conditions $\left(C_{j}\right)$ $(j=2, \ldots, n)$ do not all hold, then we are led to work with system (3). But to compute $\tilde{X}$ in (3) involves first finding the inverse to the change of variables (2),

$$
\theta=\varphi+\Phi^{*}(\varphi, x)
$$

and then calculating

$$
\tilde{X}(\varphi, x)=X\left(\varphi+\Phi^{*}(\varphi, x), x\right) .
$$

The computations of $\Phi^{*}$ and $\tilde{X}$ as power series are more difficult than those of $\Phi$ in (2) and $P$ in (19).

\section{BIBLIOGRAPHY}

1. E. Belaga, The reducibility of a system of ordinary differential equations in the neighborhood of a conditionally periodic motion, Dokl. Akad., Nauk. SSSR 143 (1962), 255-258=Soviet Math. Dokl. 3 (1962), 360-365.

2. S. P. Diliberto, Perturbation theory of periodic surfaces. I, II, III, IV, O.N.R. Reports, Univ. of California, Berkeley, 1956-1957. 
3. - Perturbation theorems for periodic surfaces, Rend. Circ. Mat. Palermo 9 (1960), $1-35$, and 10 (1961), 1-51.

4. - Perturbation theory of ordinary differential equations, Seminar notes (unpublished), Univ. of California, Berkeley, 1962-1963.

5. A. Kelley, Changes of variables near a periodic orbit, Trans. Amer. Math. Soc. 126 (1967), 316-334.

6. - The stable, center-stable, center, center-unstable, unstable manifolds; an appendix in Transversal Mappings and Flows by R. Abraham and J. Robbin, Benjamin, New York, 1967.

7. - Stability of the center-stable manifold, J. Math. Anal. Appl. 18 (1967), 336-344.

8. C. L. Siegel, Uber die Normalform analytischer Differentialgleichungen in der Nahe einer Gleichgewichtslosung, Nachr. Akad. Wiss. Göttingen Math.-Phys. K1. IIa 5 (1952), 21-30.

\section{University of CaLifornia,}

Santa Cruz, California 